\title{
RECURSIVE FIBERS OF RST ISOLS
}

\author{
T. G. MCLAUGHLIN
}

(Communicated by Andreas R. Blass)

\begin{abstract}
Motivated by a conjecture of Ellentuck concerning fibers $f_{\Lambda}^{-1}(C), f$ recursive and $C$ an element of one of Barback's "tame models" (Tame models in the isols, Houston J. Math. 12 (1986), 163-175), we study such fibers in the more general context of Nerode semirings. The principal results are that (1) all existentially complete Nerode semirings meet all of their recursive fibers, and (2) not all Nerode semirings meet all of their recursive fibers.
\end{abstract}

\section{INTRODUCTION}

Our notational and terminological conventions will be those of $[6,7]$. In particular, if $p: \omega \rightarrow \omega$ is a partial function on the natural numbers then $p_{\wedge}$ denotes the Nerode frame extension ([9] or [4, Chapter 11]) of $p$ to a partial function on the semiring $\Lambda$ of isols; similarly for $R_{\wedge}$, where $R \subseteq \omega$. A Nerode semiring [5] is a subsemiring of $\Lambda$ of the form $\mathbf{N}(X)=\left(\left\{f_{\Lambda}(X) \mid f\right.\right.$ total recursive $\},+, \cdot)$ where $X$ is a fixed but arbitrary infinite element of $\wedge$ with the property that $X \in \operatorname{domain}\left(f_{\wedge}\right)$ for all recursive $f$. Such $X$ 's have been labelled "RST isols" in the isol-theoretic literature.

Recall that by a recursive ultrapower we mean a structure for the language of Peano Arithmetic obtained as follows: let $\mathscr{F}$ be the class of unary recursive functions $f: \omega \rightarrow \omega$; let $\mathscr{B}$ be the boolean algebra of recursive subsets of $\omega$; let $\mathscr{U}$ be a nonprincipal ultrafilter in $\mathscr{B}$; for each $f \in \mathscr{F}$, let $[f]_{\mathscr{U}}$ denote the class of all those $g \in \mathscr{F}$ such that $g \equiv_{\mathscr{U}} f$, where $g \equiv_{\mathscr{U}} f$ means that $\{i \in \omega \mid g(i)=f(i)\} \in \mathscr{U}$; and let the recursive ultrapower $\mathscr{F} / \mathscr{U}$ be $\left\{[f]_{\mathscr{L}} \mid f \in \mathscr{F}\right\}$ equipped with operations of + and $\cdot$ defined in the usual ultraproduct manner. It has been noted in [5] that the Nerode semirings are, in virtue of [10], the same to within isomorphism as the (nontrivial) recursive ultrapowers and hence by [2] they are all models of $\vec{\forall} \vec{\exists}\left(\Delta_{1}^{0}\right)\left(=\Pi_{2}^{0}\right)$ Arithmetic. But every countable, nonstandard model of $\Pi_{2}^{0}$ Arithmetic is, by [2], the union of a monotone increasing chain of (isomorphic copies of) recursive ultrapowers; and by [10, Theorem 5.2], $\Lambda$ is a universal embedding structure for such models. $\Lambda$ therefore provides a modest-sized closed environment within which

Received by the editors April 15, 1991 and, in revised form, July 16, 1991.

1991 Mathematics Subject Classification. Primary 03D50; Secondary 03C65.

Key words and phrases. Nerode semiring, recursive ultrapower, existential completeness. 
to study countable $\Pi_{2}^{0}$-correct arithmetical structures, via properties of Nerode semirings.

In [1] Barback introduced what [5] shows to be a special class of Nerode semirings, namely, the "tame models". These tame models are, in fact, a subclass of the minimal Nerode semirings ( = minimal recursive ultrapowers, to within $\cong)$; this fact has been documented in [7, §3]. Each tame model per se consists exclusively of elements of $\bigwedge_{R}=$ the regressive isols, though of course arbitrary isomorphic copies of tame models, within $\Lambda$, are not in general so constituted. Moreover, all infinite elements of a tame model and, more generally, all infinite elements of an arbitrary Nerode semiring are RST isols. Barback has recently defined and shown to be nonempty a special class of tame models having the following interesting property (which amounts to saying that the model captures some portion of each of its recursive fibers):

$$
\begin{aligned}
& \text { If } f: \omega \rightarrow \omega \text { is recursive, } C \in \mathbf{N}(X) \backslash \omega \text {, and }(\exists Y \in \Lambda)\left[f_{\wedge}(Y)=C\right] \text {, } \\
& \text { then }(\exists B \in \mathbf{N}(X))\left[f_{\wedge}(B)=C\right] \text {. }
\end{aligned}
$$

(We do not bother with fibers of elements of $\omega$, since it is routine to show, using basic isol-theoretic principles, that $(\mathrm{P})$ holds if $C$ is finite.) Barback has gone on to raise (in private communication) the question whether all tame models enjoy $(P)$, indicating that it was conjectured by the late Erik Ellentuck that in fact they do. As we shall see below, this conjecture turns out to be equivalent to the conjecture that all tame models are existentially complete. We have not thus far resolved the latter question, but in $\S \S 2,3$ we show that all existentially complete Nerode semirings have property $(\mathrm{P})$ and that $(\mathrm{P})$ does not hold for arbitrary Nerode semirings. Our proof that $(\mathrm{P})$ fails for some $\mathbf{N}(X)$ is very "soft" and roundabout, giving us no insight into what such an $\mathbf{N}(X)$ might "look like".

\section{EXISTENTIAL COMPLETENESS PARTIALLY PULLS IN THE FIBER}

Readers unfamiliar with, or forgetful of, the peculiarities of $\Lambda$ may be pardoned for asking what sense the title of this section makes. Does not existential completeness simply mean that external witnesses imply internal ones? Yes, but the external witnesses have to come from extension structures that lie within the appropriate class of models, in our case the class of models of $\vec{\forall} \vec{\exists}\left(\Delta_{1}^{0}\right)$ Arithmetic. If a set of isols is a model of $\vec{\forall} \vec{\exists}\left(\Delta_{1}^{0}\right)$ Arithmetic, then (see, e.g., [6]) all of its infinite elements are RST isols; however, the isol $Y$ mentioned in the statement of property $(P)$ is not assumed to be RST. (In terms of Baire Category, most isols are not RST.) Thus our goal is to show that if $\mathbf{N}(X)$ is existentially complete (within the class of all models of $\vec{\forall} \vec{\exists}\left(\Delta_{1}^{0}\right)$ Arithmetic) and $f_{\Lambda}^{-1}(C) \neq \varnothing$, then $f_{\wedge}^{-1}(C)$ contains at least one RST isol. We shall be dealing with $\Sigma_{1}^{0}$ ultrapowers $\mathscr{P}_{\mathscr{W}} / \mathscr{W}$ (where $\mathscr{W}=$ a nonprincipal maximal filter in the class of $\Sigma_{1}^{0}$ subsets of $\omega$, and $\mathscr{P}_{\mathscr{W}}=$ the set of partial recursive functions $p(x)$ such that $\operatorname{domain}(p) \in \mathscr{W})$; these structures are existentially complete in the class of models of $\vec{\forall} \vec{\exists}\left(\Delta_{1}^{0}\right)$ Arithmetic. For general discussion of such ultrapowers, see [3, Chapter 9] (where they are called "simple models") and $[7, \S 5]$. As alluded to in [3] and stated explicitly in [7] (for a proof see [8, Lemma 2.4]), a $\Sigma_{1}^{0}$ ultrapower $\mathscr{P}_{\mathscr{W}} / \mathscr{W}$ is isomorphic to a recursive ultrapower (and hence to a Nerode semiring) iff $\mathscr{W}$ has a recursive base. 
2.1. Theorem. Every existentially complete Nerode semiring has property $(\mathrm{P})$.

Proof. Let $\mathbf{N}(X)$ be existentially complete, let $C \in \mathbf{N}(X) \backslash \omega$, let $f: \omega \rightarrow \omega$ be recursive, and suppose $Y \in \Lambda$ is such that $f_{\Lambda}(Y)=C$. By [8, Theorem 4.1], the subsemiring $\mathbf{N}(C)$ is isomorphic to a $\Sigma_{1}^{0}$ ultrapower $\mathscr{P}_{\mathscr{W}} / \mathscr{W}$ and hence is existentially complete. It therefore follows from [7, Theorem 5.18] that there is an isomorphism $\psi$ from $\mathbf{N}(C)$ onto a $\Sigma_{1}^{0}$ ultrapower $\mathscr{P}_{\mathscr{W}} / \mathscr{W}^{\prime}$ such that $\psi(C)=[\mathrm{id}]_{\mathscr{W}}$, and $\mathscr{W}^{\prime}=\left\{W \mid W\right.$ is defined in $\omega$ by a $\Sigma_{1}^{0}$ predicate $E(x)$ such that $\mathbf{N}(C) \vDash E(C)\}$. Now $\omega \vDash \forall x \forall y[f(x)=y \rightarrow y \in \operatorname{range}(f)]$; hence, by the "any relation version" [4, Theorem 18.8] of the Fundamental Nerode Metatheorem, we have that $\Lambda \vDash(\operatorname{range}(f))_{\wedge}(C)$ and hence also $\mathbf{N}(C) \vDash$ $(\operatorname{range}(f))_{\wedge}(C)$. We claim that range $(f) \in \mathscr{W}^{\prime}$. If not, then for some $W \in \mathscr{W}^{\prime}$ we have range $(f) \cap W=\varnothing$. Since $\mathscr{P}_{\mathscr{W}^{\prime}} / \mathscr{W}^{\prime}$ is isomorphic to a recursive ultrapower (in that it is isomorphic to $\mathbf{N}(C)$ ), $\mathscr{W}^{\prime}$ has, as noted above, a recursive base; i.e., $W \in \mathscr{W}^{\prime} \Rightarrow \exists R(R$ is recursive \& $R \subseteq W$ \& $R \in$ $\left.\mathscr{W}^{\prime}\right)$. So, let $R$ be a recursive element of $\mathscr{W}^{\prime}$ such that $R \subseteq W$, where $W \cap \operatorname{range}(f)=\varnothing$. By [7, Lemma 5.13] (the "Limited Los Property"), we have that $\mathscr{P}_{\mathscr{W}^{\prime}} / \mathscr{W}^{\prime} \vDash E_{R}\left([\mathrm{id}]_{\mathscr{W}^{\prime}}\right)$, where $E_{R}(x)$ is, courtesy of the Matijasevic Theorem, an existential predicate defining $R$ in $\omega$. Hence, applying $\psi^{-1}$ we have $\mathbf{N}(C) \vDash E_{R}(C)$. But $\omega \vDash \forall x\left(E_{R}(x) \rightarrow x \in R\right)$; so, by [6, Theorem 2], $\mathbf{N}(C) \vDash R_{\wedge}(C)$. But $R \cap$ range $(f)=\varnothing$, which in turn implies $R_{\wedge} \cap$ $(\text { range }(f))_{\wedge}=\varnothing$. Thus we have arrived at a contradiction. We conclude that range $(f) \in \mathscr{W}^{\prime}$. Let $M(x)$ be a $\Sigma_{1}^{0}$ predicate defining range $(f)$ in $\omega$. We clearly have $\omega \vDash \forall x(M(x) \leftrightarrow \exists y(x=f(y)))$; and $\mathscr{P}_{\mathscr{W}^{\prime}} / \mathscr{W}^{\prime} \vDash M\left([\mathrm{id}]_{\mathscr{W}^{\prime}}\right)$ by [7, Lemma 5.13] since range $(f) \in \mathscr{W}^{\prime}$. Since $\mathscr{P}_{\mathscr{W}^{\prime}} / \mathscr{W}^{\prime} \vDash \vec{\forall} \vec{\exists}\left(\Delta_{1}^{0}\right)$ Arithmetic, we get $\mathscr{P}_{\mathscr{W}^{\prime}} / \mathscr{W}^{\prime} \vDash \exists y\left([\text { id }]_{\mathscr{W}}=f(y)\right)$. Hence, applying $\psi^{-1}$, we have that $\mathbf{N}(C) \vDash \exists y\left(C=f^{\mathbf{N}(C)}(y)\right)$. But therefore also $\mathbf{N}(X) \vDash \exists y\left(C=f^{\mathbf{N}(X)}(y)\right)$, since [2, Theorem 1.8] $f^{\mathrm{N}(C)}=\left.f^{\mathbf{N}(X)}\right|_{\mathbf{N}(C)}$. By [6, Lemma 2], however, $f^{\mathbf{N}(X)}=$ $\left.f_{\wedge}\right|_{\mathbf{N}(X)}$, whence $\mathbf{N}(X) \vDash \exists Y\left(C=f_{\wedge}(Y)\right)$.

\section{THERE EXIST $\mathbf{N}(X)$ 'S FOR WHICH (P) FAILS}

3.1. Theorem. If all Nerode semirings have property $(\mathrm{P})$ then they are all existentially complete.

Proof. Let $\mathbf{N}(X)$ be a Nerode semiring, and let $\mathscr{F} / \mathscr{U}$ be a recursive ultrapower such that $\psi: \mathbf{N}(X) \rightarrow \mathscr{F} / \mathscr{U}$ is an isomorphism, with $\psi(X)=[\text { id }]_{\mathscr{U}}[7$, Theorem 2.13]. It suffices to show that $\mathscr{F} / \mathscr{U}$ is existentially complete. Let $\mathscr{M}$ be a countable model of $\vec{\forall} \vec{\exists}\left(\Delta_{1}^{0}\right)$ Arithmetic such that $\mathscr{F} / \mathscr{U} \subseteq \mathscr{M}$, and suppose that $\exists y R\left(y, c_{0}, \ldots, c_{k}\right)$ holds in $\mathscr{M}$, where $R$ is $\Delta_{1}^{0}$ and $c_{0}, \ldots, c_{k} \in$ $\mathscr{F} / \mathscr{U}$. Now we observe that there is a recursive function $g(y)$ such that $\omega \vDash \forall x_{0} \cdots \forall x_{k}\left[\exists y R\left(y, x_{0}, \ldots, x_{k}\right) \leftrightarrow \exists y\left(g(y)=2^{x_{0}+1} \cdots p_{k}^{x_{k}+1}\right)\right],\left\{p_{j}\right\}$ being the sequence of primes in order of magnitude. Thus, if $\exists y R\left(y, x_{0}, \ldots, x_{k}\right)$ defines $\omega^{k+1}$ we may take $g(y) \stackrel{\text { df }}{=} 2^{(y)_{0}+1} \cdots p_{k}^{(y)_{k}+1}$ for all $y$. Otherwise, we define

$$
g(y)= \begin{cases}2^{x_{0}+1} \cdots p_{k}^{x_{k}+1} & \text { if } y=2^{z} 3^{x_{0}+1} \cdots p_{k+1}^{x_{k}+1} \& R\left(z, x_{0}, \ldots, x_{k}\right), \\ p_{k+1}, & \text { otherwise. }\end{cases}
$$

Since $\mathscr{M} \vDash \exists y R\left(y, c_{0}, \ldots, c_{k}\right)$, we have that $\mathscr{M} \vDash \exists y\left(g(y)=2^{c_{0}+1} \cdots p_{k}^{c_{k}+1}\right)$. 
Now, by [10], there is an isomorphism $\tau$ from $\mathscr{M}$ onto an $\vec{\forall} \vec{\exists}\left(\Delta_{1}^{0}\right)$-correct subsemiring $\mathscr{S}$ of $\Lambda$. Consider $\tau(\mathscr{F} / \mathscr{U})$ : if $X_{0}=\tau\left([\text { id }]_{\mathscr{U}}\right)$, it follows from [6, Lemma 2] that $\tau(\mathscr{F} / \mathscr{U})=\mathbf{N}\left(X_{0}\right)$. But thus $\mathbf{N}\left(X_{0}\right)$ is an isomorphic copy of $\mathscr{F} / \mathscr{U}$ contained in $\mathscr{S}, \mathscr{S} \vDash \exists y\left(g(y)=2^{\tau\left(c_{0}\right)+1} \cdots p_{k}^{\tau\left(c_{k}\right)+1}\right)$, and, again by [6, Lemma 2], $g^{\mathscr{S}}(Y)=g_{\wedge}(Y)$ for any $Y \in \mathscr{S}$. Thus, for some $Y \in \mathscr{S}$ we have $g_{\wedge}(Y)=2^{\tau\left(c_{0}\right)+1} \cdots p_{k}^{\tau\left(c_{k}\right)+1} \in \mathbf{N}\left(X_{0}\right)$. Since we are assuming $\mathbf{N}\left(X_{0}\right)$ has property $(\mathrm{P})$, this implies that $g_{\wedge}(Z)=2^{\tau\left(c_{0}\right)+1} \cdots p_{k}^{\tau\left(c_{k}\right)+1}$ for some $Z \in \mathbf{N}\left(X_{0}\right)$. So, pulling matters back to $\mathscr{F} / \mathscr{U}$ via $\tau^{-1}$, we see that $\mathscr{F} / \mathscr{U} \vDash \exists y(g(y)=$ $\left.2^{c_{0}+1} \ldots p_{k}^{c_{k}+1}\right)$ and hence $\mathscr{F} / \mathscr{U} \vDash \exists y R\left(y, c_{0}, \ldots, c_{k}\right) . \mathscr{F} / \mathscr{U}$, therefore, is existentially complete, and we are done.

3.2. Corollary. There exist Nerode semirings $\mathbf{N}(X)$ such that $\mathbf{N}(X)$ fails to have property $(\mathrm{P})$.

Proof. It is well known (see, e.g., [3, Lemma 1.10; 2, Theorem 3.2]) that not all recursive ultrapowers, hence not all Nerode semirings, are existentially complete.

3.3. Remarks. As we noted in $\S 1$, our proof of Corollary 3.2 is distressingly indirect: we have not even come close to "constructing" an example (unless the "compactness" arguments of [10], on which Nerode's isomorphism theorems are based, are viewed as constructive). Taken together, Theorems 2.1 and 3.1 are rather strongly suggestive of the possibility that property $(P)$ is in fact equivalent to existential completeness, for the class of Nerode semirings. However, as we shall prove in Theorem 3.4, this is not the case. We observe that Theorem 3.1 remains true (and by essentially the same proof) if in its statement we replace the class of Nerode semirings by the class of tame models. But while it is rather easy to construct existentially complete tame models, we do not know at present how to construct any that are not existentially complete. In light of these various observations, we raise the following two questions. (1) How might the proof of Corollary 3.2 be made more constructive? (2) Are all tame models existentially complete? The (presumably) special class of tame models recently introduced by Barback is the class of those he terms "strongly torre," defined by the property of having a "strongly torre generator," i.e., an infinite isol $X \in \bigwedge_{R}$ such that for every $\Sigma_{1}^{0}$ subset $E$ of $\omega$, there are recursive sets $R_{1}$ and $R_{2}$ so that (i) $X \in E_{\wedge} \cup(\omega \backslash E)_{\wedge}$, (ii) $X \in E_{\wedge} \Rightarrow\left(R_{1} \subseteq E \& \quad X \in\left(R_{1}\right)_{\wedge}\right)$, and (iii) $X \in(\omega \backslash E)_{\wedge} \Rightarrow\left(R_{2} \subseteq \omega \backslash E \& X \in\left(R_{2}\right)_{\wedge}\right)$. It is not hard to prove, using the techniques of the present paper, that a tame model is strongly torre $\Leftrightarrow$ it is existentially complete; thus, question (2) can be rephrased to ask whether all tame models are strongly torre. Somehow this strikes us as unlikely.

\subsection{Theorem. (P) is not preserved under $(+, \cdot)$-isomorphisms within $\bigwedge$.}

Proof. Let $\mathbf{N}\left(X_{0}\right)$ be a Nerode semiring not having property (P). We observe that a careful reading of the proof of [10, Theorem 5.3] reveals that the result applies not just to models of true arithmetic, but in fact to all countable models of $\vec{\forall} \vec{\exists}\left(\Delta_{1}^{0}\right)$ Arithmetic. So let $\mathscr{S}$ be a subsemiring of $\Lambda$ such that $\psi: \mathbf{N}\left(X_{0}\right) \rightarrow \mathscr{S}$ is an isomorphism with the following property: if $R \subseteq \omega$ is $\Sigma_{1}^{0}$ and $\exists_{R}(x)$ is an existential predicate defining $R$ in $\omega$, then $\mathscr{S} \vDash R_{\wedge}(X)$ if and only if $\mathbf{N}\left(X_{0}\right) \vDash \exists_{R}\left(\psi^{-1}(X)\right)$, for every $X \in \mathscr{S}$. We claim that $\mathscr{S}$ 
has property (P). For suppose $C \in \mathscr{S} \backslash \omega, Y \in \Lambda$, and $f_{\wedge}(Y)=C, f$ recursive. As in the proof of Theorem 2.1, $\wedge \vDash(\operatorname{range}(f))_{\wedge}(C)$, so that $\mathscr{S} \vDash(\operatorname{range}(f))_{\wedge}(C)$. So $\mathbf{N}\left(X_{0}\right) \vDash \exists_{\operatorname{range}(f)}\left(\psi^{-1}(C)\right)$. Hence, $\psi$ being an isomorphism, $\mathscr{S} \vDash \exists_{\text {range }(f)}(C)$. But $\omega \vDash \forall x\left[\exists_{\text {range }(f)}(x) \leftrightarrow \exists y(f(y)=x)\right]$; so $\mathscr{S}$ also satisfies this sentence and, therefore by [6, Theorem 2] we have $\mathscr{S} \vDash \exists Y\left(f_{\wedge}(Y)=C\right)$.

3.5. Corollary. Property $(\mathrm{P})$ does not characterize existential completeness for Nerode semirings.

Proof. Existential completeness is an isomorphism invariant.

\section{SOME RELATED OBSERVATIONS}

There is a certain amount of peripheral information to be gleaned from the considerations of $\S \S 2,3$. Let us begin with a proof that every $\mathbf{N}(X)$ does satisfy a suitably weakened version of $(\mathrm{P})$.

4.1. Theorem. Let $\left(\mathrm{P}^{\prime}\right)$ denote property $(\mathbf{P})$ with " $f: \omega \rightarrow \omega$ is recursive" replaced by " $f: \omega \rightarrow \omega$ is recursive \& $\operatorname{range}(f)$ is recursive." Then every Nerode semiring has property $\left(\mathbf{P}^{\prime}\right)$.

Proof. It will suffice to apply a straightforward modification of a fragment of the proof given for Theorem 2.1. Let $f$ be a unary recursive function having recursive range; and let $\mathbf{N}(X)$ be a Nerode semiring and $C$ an element of $\mathbf{N}(X) \backslash \omega$ such that $(\exists Y \in \Lambda)\left(f_{\wedge}(Y)=C\right)$. As in the proof of Theorem 2.1, we have $\mathbf{N}(C) \vDash(\operatorname{range}(f))_{\wedge}(C)$. But $\operatorname{range}(f)$ is recursive; and we have that $\omega \vDash \forall x\left(x \in \operatorname{range}(f) \leftrightarrow R_{\text {range }(f)}(x)\right)$, where $R_{\text {range }(f)}(x)$ is an existential predicate defining range $(f)$ in $\omega$. It follows from [6, Theorem 2] that $\mathbf{N}(C) \vDash R_{\text {range }(f)}(C)$. But $\omega \vDash \forall x\left[R_{\text {range }(f)}(x) \leftrightarrow \exists y(x=f(y))\right]$; so, again by [6, Theorem 2], we have $\mathbf{N}(C) \vDash \exists Y\left(C=f_{\wedge}(Y)\right)$. Since $\mathbf{N}(C) \subseteq \mathbf{N}(X)$, we also have $\mathbf{N}(X) \vDash(\exists Y)\left(C=f_{\wedge}(Y)\right)$, and the proof is complete.

Next we note that it is well known among people experienced in isol theory that $\Lambda \vDash E_{\wedge}(X)$ does not, in general, imply $\exists R(R$ is recursive, $R \subseteq E$, and $\left.\Lambda \vDash R_{\wedge}(X)\right)$, where $E$ is a $\Sigma_{1}^{0}$ predicate. It is not, however, easy to track down explicit renderings of this fact in the literature. So, we include among our "side results" the following:

4.2. Theorem (Folklore). There exist $\Sigma_{1}^{0}$ subsets $E$ of $\omega$ and isols $X$, such that (1) $\wedge \vDash E_{\wedge}(X)$ and (2) there is no recursive set $R \subseteq E$ for which $\Lambda \vDash$ $R_{\wedge}(X)$.

Proof. Choose a maximal (nonprincipal) $\Sigma_{1}^{0}$ filter $\mathscr{W}$ so that $\mathscr{W}$ does not have a recursive base. (The "standard" way to do this is noted in [3, Chapter 9, p. 158].) Then $\mathscr{P}_{\mathscr{W}} / \mathscr{W} \vDash \vec{\forall} \vec{\exists}\left(\Delta_{1}^{0}\right)$ Arithmetic; and, by [10], there is an isomorphism $\tau: \mathscr{P}_{\mathscr{W}} / \mathscr{W} \rightarrow \mathscr{M}$ from $\mathscr{P}_{\mathscr{W}} / \mathscr{W}$ onto a countable, $\vec{\forall} \vec{\exists}\left(\Delta_{1}^{0}\right)$ correct subsemiring $\mathscr{M}$ of $\Lambda$. Since $\mathscr{W}$ has no recursive base, we may choose $W \in \mathscr{W}$ so that $R \notin \mathscr{W}$ for all recursive $R \subseteq W$. By [7, Lemma 5.13], $\mathscr{P}_{\mathscr{W}} / \mathscr{W} \vDash E_{W}\left([\mathrm{id}]_{\mathscr{W}}\right)$ where $E_{W}(x)$ is a $\Sigma_{1}^{0}$ predicate defining $W$ in $\omega$. Hence $\mathscr{M} \vDash E_{W}\left(\tau\left(\left[\mathrm{id}_{\mathscr{W}}\right)\right)\right.$. By the Matijasevic Theorem, we may assume that $E_{W}$ is existential and indeed diophantine in form. But then the sentence $\forall x\left(E_{W}(x) \rightarrow x \in W\right)$ is equivalent to a Horn sentence (containing 
$W$ as a predicate) that is true in $\omega$. So by [4, Theorem 18.8], we have that $\mathscr{M} \vDash W_{\wedge}\left(\tau\left([\mathrm{id}]_{\mathscr{W}}\right)\right)$ and hence $\Lambda \vDash W_{\wedge}\left(\tau\left([\mathrm{id}]_{\mathscr{W}}\right)\right)$. But, we claim, $R_{\wedge}\left(\tau\left([\mathrm{id}]_{\mathscr{W}}\right)\right)$ cannot hold in $\Lambda$ if $R$ is a recursive subset of $W$. For if $R_{\wedge}\left(\tau\left([\mathrm{id}]_{\mathscr{W}}\right)\right.$ did hold for such an $R$, then, letting $E_{R}(x)$ be an existential predicate defining $R$ in $\omega$, we would have by [6, Theorem 2] that $\mathscr{M} \vDash E_{R}\left(\tau\left([\text { id }]_{\mathscr{W}}\right)\right)$ and hence $\mathscr{P}_{\mathscr{W}} / \mathscr{W} \vDash E_{R}\left([\mathrm{id}]_{\mathscr{W}}\right)$. But then, by [7, Lemma 5.13], $R \in \mathscr{W}$-a contradiction. The proof is complete.

Finally, noting that we have applied [6, Theorem 2] a number of times in the foregoing discussion, we ask whether that theorem might be improved to take $\Sigma_{1}^{0}$ predicates into account as well as $\Delta_{1}^{0}$ ones. The statement of [6, Theorem 2 ] is that if the language $L$ of first-order arithmetic is replaced by the richer language $L_{N}$, the "Nerode language for isol theory", then any $\vec{\forall} \vec{\exists}$ sentence of $L_{N}$ that is true in $\omega$ is true also in $\mathscr{M}, \mathscr{M}$ any set of isols that models all the $\omega$-true $\vec{\forall} \vec{\exists}$ sentences of $L$. To be more specific about $L_{N}$, the new symbols it contains are: a relation constant $R_{n, i}$ for each $n$-ary recursive relation, and a function symbol $f_{n, i}$ for each $n$-ary almost recursive combinatorial function. (Since any recursive function is the difference of two recursive combinatorial functions, it is all right to assume that, in addition, $L_{N}$ contains symbols for all the recursive functions.) What we are asking is: can the recursive relations, in the above statement of [6, Theorem 2], be expanded to the $\Sigma_{1}^{0}$ relations? The answer is no.

4.3. Theorem. Theorem 2 of [6] is false if the class of recursive relations on $\omega$ is enlarged to the class of recursively enumerable relations on $\omega$.

Proof. Applying Corollary 3.2, let $\mathbf{N}(X)$ be a Nerode semiring that does not have property $(\mathbf{P})$. Let $Y \in \Lambda$ and $f$ (recursive) be such that $f_{\wedge}(Y)=C \in$ $\mathbf{N}(X)$, but no such $Y$ lies in $\mathbf{N}(X)$. Now, as in the proof of Theorem 2.1, $\mathbf{N}(X) \vDash(\operatorname{range}(f))_{\wedge}(C)$. If, then, [6, Theorem 2] were generalizable in the way indicated, we would have $\mathbf{N}(X) \vDash E_{\text {range }(f)}(C)$ where $E_{\text {range }(f)}(x)$ is an existential predicate defining range $(f)$ in $\omega$. But $\omega \vDash \forall x\left[E_{\text {range }(f)}(x) \leftrightarrow \exists y(f(y)=\right.$ $x)$ ], whence $\mathbf{N}(X) \vDash \exists Y\left(f_{\wedge}(Y)=C\right)$-a contradiction.

4.4. Remarks. (a) It is possible to prove Theorems 2.1 and 4.1 in a way that could perhaps be considered slightly more "constructive", by putting more emphasis on the limited Los property. For example, in proving 2.1, once we know that range $(f) \in \mathscr{W}^{\prime}$ we can define a recursive function $h(x)$ in such a way that $\{i \mid f(h(i))=i\} \in \mathscr{W}^{\prime}$, and then note that $f_{\wedge}\left(\psi^{-1}\left([h]_{\mathscr{W}^{\prime}}\right)\right)=\psi^{-1}\left([\mathrm{id}]_{\mathscr{W}^{\prime}}\right)$.

(b) Suppose $f_{\wedge}(Y)=C \in \mathbf{N}(X) \backslash \omega$, where $f$ is recursive. If one did not know about Corollary 3.2, one might attempt to prove Theorem 2.1 for all $\mathbf{N}(X)$ by attempting to first replace $f$ by a recursive function $f^{*}$ with recursive range so that $f_{\wedge}^{*}(Y)=f_{\wedge}(Y)$, then getting $B \in \mathbf{N}(X)$, via 4.1 , so that $f_{\wedge}^{*}(B)=$ $f_{\wedge}^{*}(Y)=C$, and finally showing that (at least for suitable $f^{*}$ ) this $B$ can be chosen so that $f_{\Lambda}^{*}(B)=f_{\wedge}(B)$. But 3.2 implies that either the first or the third step in this program cannot be carried out.

(c) Theorem 4.3 (or rather its proof) provides a solution, in the negative, to the last of the problems posed at the end of the author's paper Embeddings of and into Nerode semirings, Israel J. Math. 60 (1987), 65-88. That problem was whether, in general, $\mathbf{N}(X) \vDash \forall x(x \in R \leftrightarrow \Sigma(x))$, where $R$ is a $\Sigma_{1}^{0}$ subset of $\omega$ and $\Sigma(x)$ is a $\Sigma_{1}^{0}$ predicate defining it in $\omega$. 


\section{REFERENCES}

1. J. Barback, Tame models in the isols, Houston J. Math. 12 (1986), 163-175.

2. J. Hirschfeld, Models of arithmetic and recursive functions, Israel J. Math. 20 (1975), 111126.

3. J. Hirschfeld and W. Wheeler, Forcing, arithmetic, and division rings, Lecture Notes in Math., vol. 454, Springer, Berlin, 1975.

4. T. McLaughlin, Regressive sets and the theory of isols, Lecture Notes in Pure and Appl. Math., vol. 66, Marcel Dekker, New York, 1982.

5. __ Nerode semirings and Barback's 'tame models,' Houston J. Math. 12 (1986), 211-223.

6. $\ldots$, Some properties of $\forall \exists$ models in the isols, Proc. Amer. Math. Soc. 97 (1986), 495502.

7. __ Sub-arithmetical ultrapowers: a survey, Ann. Pure Appl. Logic 49 (1990), 143-191.

8. __ Recursive ultrapowers, simple models, and cofinal extensions, Arch. Math. Logic 31 (1992).

9. A. Nerode, Extensions to isols, Ann. of Math. (2) 73 (1961), 362-403.

10. _ Diophantine correct nonstandard models in the isols, Ann. of Math. (2) 84 (1966), 421-432.

Department of Mathematics, Texas Tech University, Lubbock, Texas 79409

E-mail address: A1YSP@TTU.edu 